Research Article

\title{
Morphological and Mechanical Properties of Poly (Butylene Terephthalate)/High-Density Polyethylene Blends
}

\author{
Nga Thi-Hong Pham (i) and Van-Thuc Nguyen \\ Mechanical Engineering Faculty, HCMC University of Technology and Education, 1 Vo Van Ngan St. Thu Duc District, \\ HCM City 71307, Vietnam
}

Correspondence should be addressed to Nga Thi-Hong Pham; hongnga@hcmute.edu.vn

Received 25 September 2020; Revised 9 November 2020; Accepted 2 December 2020; Published 14 December 2020

Academic Editor: Hongchao Kou

Copyright (c) 2020 Nga Thi-Hong Pham and Van-Thuc Nguyen. This is an open access article distributed under the Creative Commons Attribution License, which permits unrestricted use, distribution, and reproduction in any medium, provided the original work is properly cited.

\begin{abstract}
Poly (butylene terephthalate) (PBT) is a popular thermoplastic polyester resin but has low strength and low melting point. To improve its properties, PBT is often mixed with other resins, such as high-density polyethylene (HDPE). In this study, PBT/HDPE samples with 100\% PBT, 5\%, 10\%, 15\%, and 100\% HDPE are generated and tested. The samples are analyzed by tensile strength, flexural strength, impact strength, and SEM tests. Adding HDPE will reduce tensile strength compared to pure PBT, in which 5\%, $10 \%$, and 15\% PBT/HDPE samples obtain the values $40.23,38.11$, and $27.77 \mathrm{MPa}$, respectively. These values are lower than that of pure PBT but still higher than that of HDPE. Improving the HDPE portion mostly results in decreasing flexural strength. The flexural strengths of these samples are $87.79,70.47,55.3,58.98$, and $19.14 \mathrm{MPa}$ corresponding to $100 \% \mathrm{PBT}, 5 \%, 10 \%, 15 \%$, and $100 \%$ HDPE samples, respectively. Moreover, the SEM microstructure of PBT and HDPE indicates a two-phase heterogeneous mixture with little or no adhesion between these phases.
\end{abstract}

\section{Introduction}

Poly (butylene terephthalate) (PBT) is an important thermoplastic with good properties of chemical resistance, insulation, lubrication, and processing and has been widely used in the automobile and construction industry. It exhibits high rates of crystallization, allowing short cycle times when pressing. However, its tensile strength and impact resistance is rather low. To improve its characteristics, PBT can be mixed with other polymers.

Polymer blending is a popular and efficient method in which new materials can be produced to meet specific and diverse demands. Blended polymers are preferred over homogeneous types because they can perform the advantages of each component. For instance, semicrystalline polybutylene terephthalate (PBT) has some disadvantages such as low impact strength and low melting strength. Therefore, it can be mixed with a variety of polyethylene polymers, such as high-density polyethylene (HDPE), lowdensity polyethylene (LDPE), linear low-density polyethylene (LLDPE), and polypropylene (PP), to create thermoplastic materials that are commonly used in the packaging industry. The blended polymers also provide an alternative means of using recycled plastic waste by mixing one to another type of polymer.

Toothbrush bristles are often made from polyamide (PA) and PBT fiber mixture. To recycle, this mixture must be classified into PA fiber and PBT fiber. After separation, PA is often reused due to its high cost $[1,2]$. PBT is a widely used thermoplastic polyester resin, but it still has certain disadvantages such as low strength, low melting point, and low durability. PBT is often mixed with other resins to improve its characteristics. However, mixing two or more polymers together often leads to incompatibilities. In addition, Jubinville et al. [3] showed that the mixture can also be well dispersed by using a suitable compatibilizer. 
According to Greiner et al. [4], the mixture of PBT and PC can be sintering by a liquid-phase method by applying selective laser sintering (SLS). Wang et al. [5] investigated HDPE with a rigid PA-6/polybutylene terephthalate core. It was found that the strength of the sample of a mixture of three different substances, polyethylene PA-6/PBT (80/10/ 10), was $43.87 \mathrm{~kJ} / \mathrm{m}^{2}$, two times higher than pure HDPE. Microstructure observations show that the addition of polybutylene terephthalate and PA-6 leads to the appearance of core-dispersed particles in an HDPE matrix, which is the main reason for HDPE hardening. In a study by Chalabi Tehran et al. [6], thermoplastic polyurethane (TPU) and clay nanoparticles have been integrated into PBT to improve impact properties and tensile strength. PBT/TPU samples $(90 / 10,80 / 20$, and 70/30) were prepared by melt mixing using a coaxial twin-screw extruder, followed by injection molding. In the next stage, clay nanoparticles with different weight fractions were added to the PBT/TPU (80/20) mixture. SEM pictures illustrate good compatibility between TPU and PBT. The addition of TPU to PBT significantly increases impact strength but reduces tensile strength and elastic modulus. The incorporation of clay nanoparticles into a PBT/TPU mixture has greatly improved the tensile properties. A balance of tensile and impact characteristics has been found in the PBT/TPU/clay nanocomposite system (80/20/3). Salmoria et al. [7] investigated the structure and properties of blended samples PA-12 and PBT. Higher values for the bending modulus and tensile strength expressed by the specimen 90/10 PA12/PBT show the hardening effect of the PBT particles in the PA-12 matrix. These results indicate the potential application in the manufacture of 90/10 PA-12/PBT with high stiffness and fatigue resistance. Yanping et al. [8] researched the PBT/TPU mixture prepared by melting their mixture. The flexibility, crystalline behavior, and hardening mechanism of PBT/TPU mixtures have been studied. The results have proved that adding TPU will help improve the toughness of the mixture.

The development of microheterogeneous composites with a structural discontinuity is becoming one of the important trends in the production of new synthetic polymer materials. The mixture of two compatible polymers can increase the characteristics of the composite. A popular composite polymer-polymer system is PBT and polyolefin resins [9-11]. Studies of PBT and polyolefin-based composites have shown significant improvement in their physical and mechanical properties when adding $2-5 \mathrm{wt} \%$ PBT. Differences in tensile values for PBT and PBT/polyolefin samples were explained by forming a loose-packed interfacial layer (LIL) between thermodynamically incompatible polymers, where crystal formation is not possible. Under high temperatures and deformation, the molecular weight distribution of polymers may change as certain low-molecular fractions can be formed, contributing to the plasticizer process [12,13]. Mikitaev et al. [9] have proved that HDPE is one of the most promising materials that can improve the properties of PBT. However, $\mathrm{PBT} / \mathrm{HDPE}$ is an incompatible mixture because of the lack of bonding on the surface of these two materials, thereby leading to its poor mechanical properties.

\section{Materials and Methods}

The experiment chose to use HDPE resins from SABICHDPE 4024, Saudi Arabia, which was provided by Thuan Thang Plastic Co., Ltd. PBT was taken from the brush hair scraps, and then PBT resins were created from the bristles. Samples of HDPE and PBT resins were mixed and pressed according to the proportions shown in Table 1.

2.1. Plastic Drying. The samples are dried for $10 \mathrm{~h}$ at $80^{\circ} \mathrm{C}$ before pressing by the plastic injection molding machine.

2.2. Tensile Testing. The samples are tested following the ASTM D638 standard. The number of tensile test samples included 24 samples divided into 6 groups. The sample tested must be free of sharp edges, oil, grease, and other impurities. Before testing, the samples are stored in an environment with a temperature of $23 \pm 2^{\circ} \mathrm{C}$ and a humidity of $50 \pm 5 \%$, for at least 40 hours.

The dimensions of the samples are shown in Figure 1. The sample is mounted on the testing machine as shown in Figure 2 and pulled at a speed of $50 \mathrm{~mm} / \mathrm{min}$ at room temperature.

The test is performed on the universal Shimadzu Autograph AG-X Plus machine. The expansion meter is a highresolution camera $(1.8 \mu \mathrm{m})$ that does not contact with the sample. The basic specifications of the testing machine are maximum test power of $20 \mathrm{kN}$, speed range of $0.001-1600 \mathrm{~mm} / \mathrm{min}$, speed accuracy of $\pm 0.1 \%$ of test speed, operating environment at $5-50^{\circ} \mathrm{C}$, and data collection rate of $1000 \mathrm{~Hz}$.

2.3. Flexural Testing. The samples are tested following the ISO 178 standard as shown in Figure 3 and Figure 4. The number of samples is 30 divided into 6 groups.

2.4. Izod Impact Testing. The samples are tested following the ASTM D256 standard (Figure 5). According to ASTM D256, we need to check at least 4 samples for each sample group to achieve high accuracy. However, to prevent the sample from being damaged during measurement, 7 samples are taken for each group of samples tested. Therefore, the total number of samples is 42 . The test is performed on the impact tester Tinius Olsen IT504 (Figure 6).

2.5. Microstructure Examination. To observe the sample microstructure, the experiment uses a high-resolution scanning electron microscope (SEM) (HITACHI S-4800).

\section{Results and Discussion}

3.1. Surface Morphology. Figure 7 shows the specimen of the tensile test, flexural strength test, and Charpy impact test. Through the practice of injection molding, it can be seen that sample $\mathrm{H} 0$ is not easy to inject, while sample $\mathrm{H} 100$ has a high shrinkage rate. Samples H5, H10, and H15 have a good surface quality. 
TABLE 1: Composition of the testing sample.

\begin{tabular}{lcc}
\hline Samples & & Component (wt.\%) \\
\hline- & PBT & HDPE \\
H0 & 100 & 0 \\
H5 & 95 & 5 \\
H10 & 90 & 10 \\
H15 & 85 & 15 \\
H100 & 0 & 100 \\
\hline
\end{tabular}

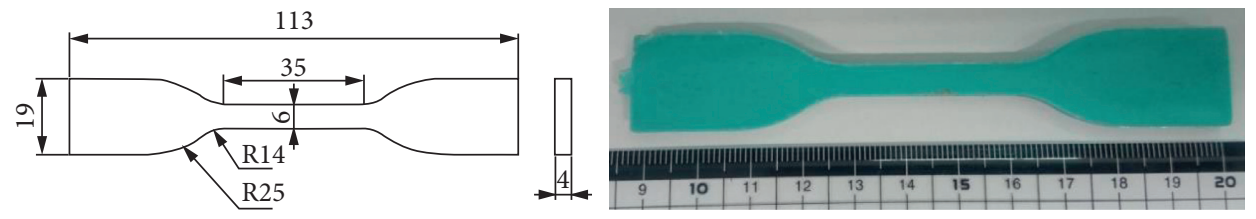

Figure 1: Sample size of the tensile test.

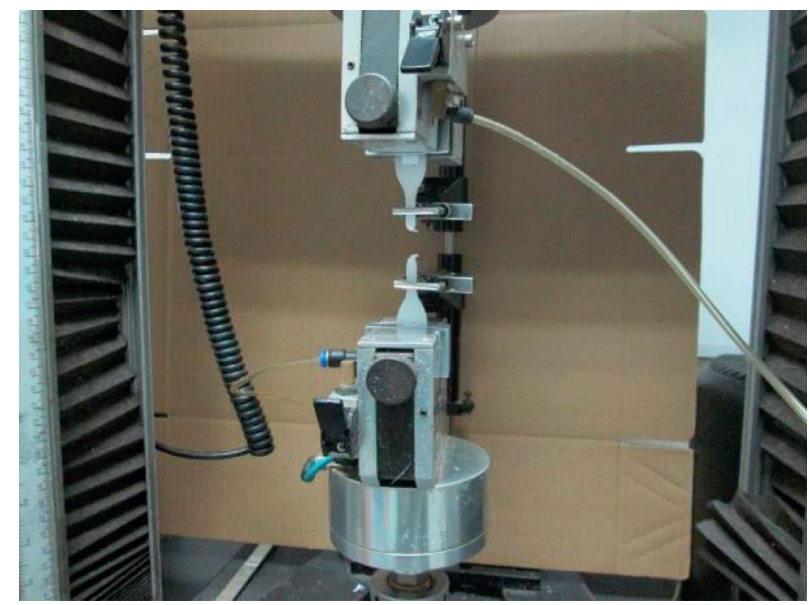

Figure 2: Sample mounted on the universal Shimadzu Autograph AG-X Plus machine.

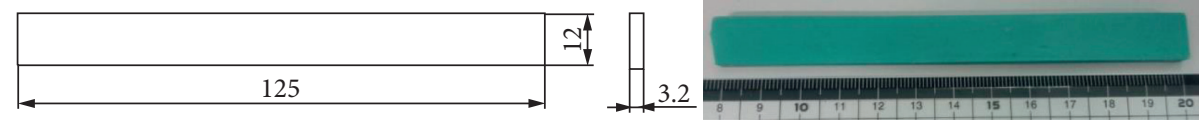

FIGURE 3: Sample size for bending strength testing.

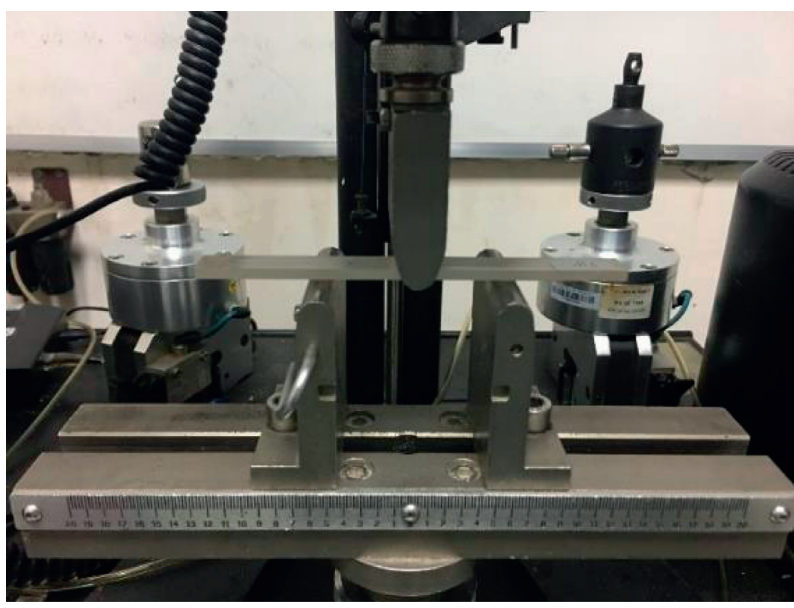

FIGURE 4: Sample mounted on the Instron 5566 machine. 

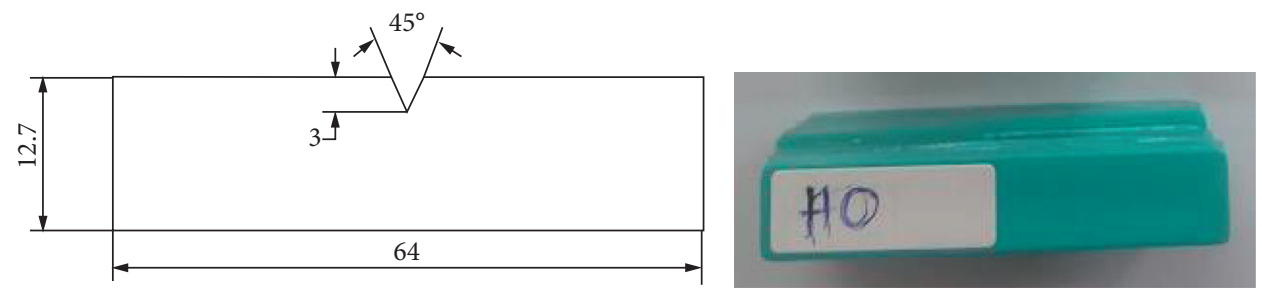

Figure 5: Sample size for Izod impact strength.

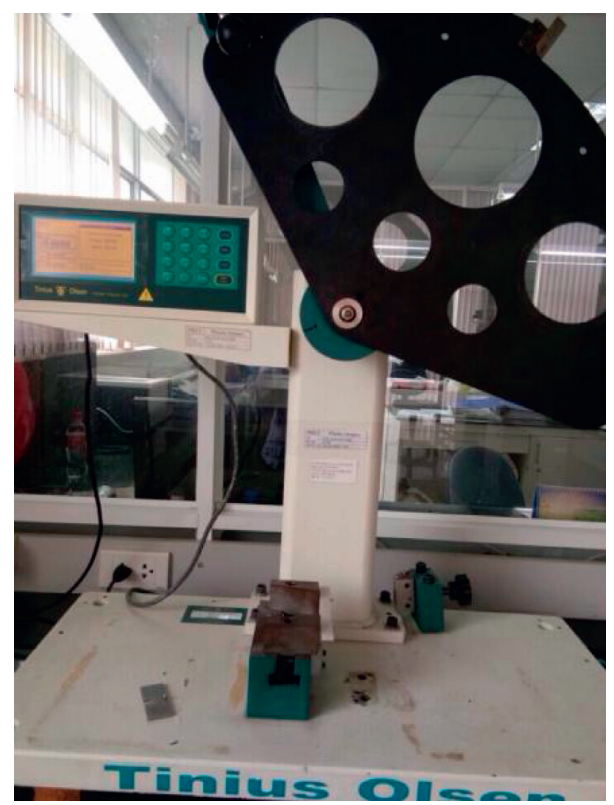

Figure 6: Izod impact tester Tinius Olsen IT504.

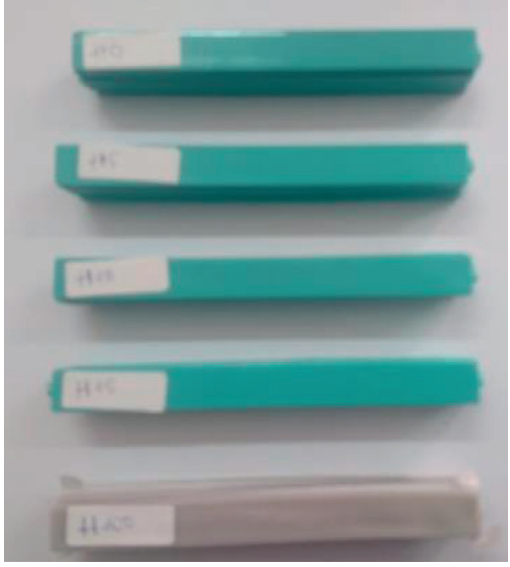

(a)

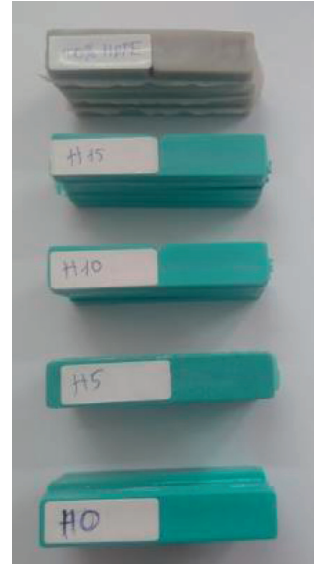

(b)

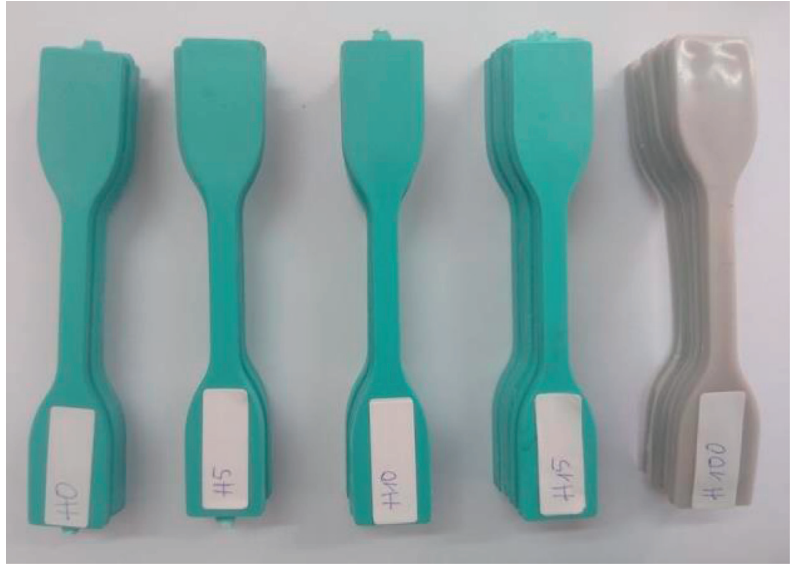

(c)

Figure 7: Specimen of the tensile test, flexural strength test, and Charpy impact test: (a) flexural strength samples, (b) Charpy impact samples, and (c) tensile test samples.

Figure 8 presents the stress-strain curves of the samples. The pure PBT is very brittle with a very low value of elongation of about $2.5 \%$. Adding HDPE to PBT will lead to a decrease in tensile strength. The tensile strength values of the composite samples are in the middle of pure PBT and HDPE.
Generally, the fracture mechanism of these samples is also brittle fracture.

Figure 9 describes the effect of the HDPE content on the tensile strength of the PBT/HDPE mixture. Samples H5, H10, and H15 have the values 40.23, 38.11, and $27.77 \mathrm{MPa}$, 


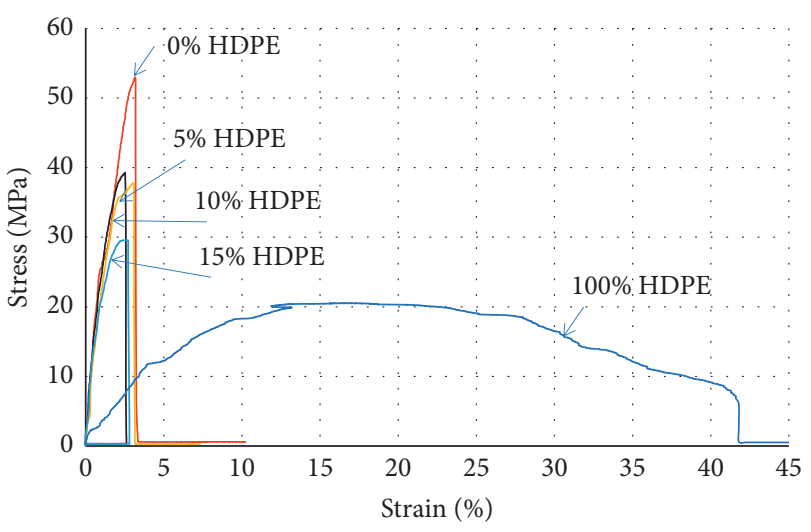

FIGURE 8: Stress-strain curves of samples.

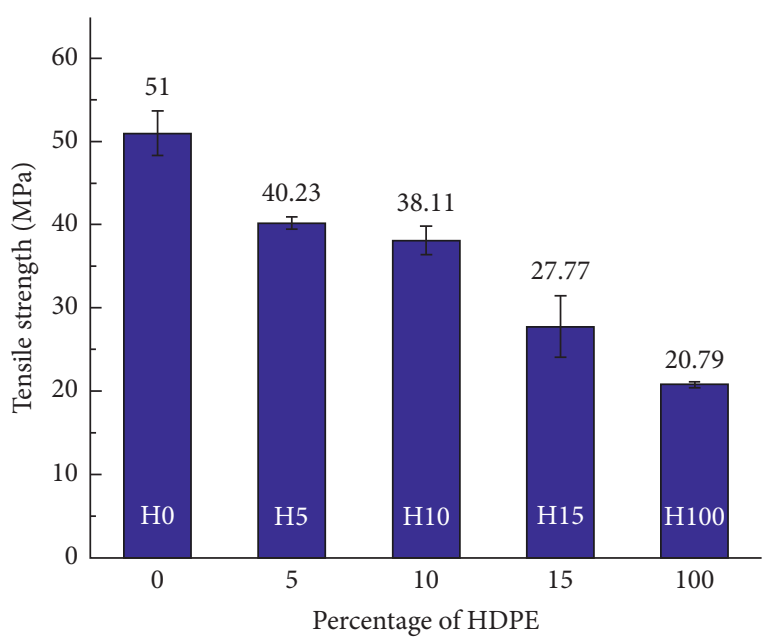

FIGURE 9: Effect of HDPE percentage on the tensile strength of the PBT/HDPE blends.

respectively. These values are lower than the $51.00 \mathrm{MPa}$ value of pure PBT but higher than that of HDPE which is $20.79 \mathrm{MPa}$. In general, adding HDPE will reduce tensile strength compared to pure PBT. Because, for incompatible blends such as PBT/HDPE, incompatibility between the two components can result in weaker bonding between the components, poorer mechanical properties, and other problems such as welding line failure in the injection molding process.

The average elongation values at break are shown in Figure 10. The mixtures of $0-15 \%$ HDPE have a low value of elongation, vibrating about $2.5 \%$. Therefore, by adding $0-15 \%$ HDPE to the mixture, the elongation of the sample does not cause appreciable change. The pure HDPE sample achieves the highest value of elongation of $24.09 \%$. The HDPE particles are dispersed in the PBT/HDPE matrix. In this matrix, the two phases are almost co-continuous, creating higher barrier properties for toluene than pure PE [12].

Figure 11 shows the stress curves of the flexural test of the PBT/HDPE mix. Similar to the tensile strength, initially, the flexural stress of these blended samples gradually increases, followed by a sudden decrease when the samples are destroyed. However, with a pure HDPE sample, the stress

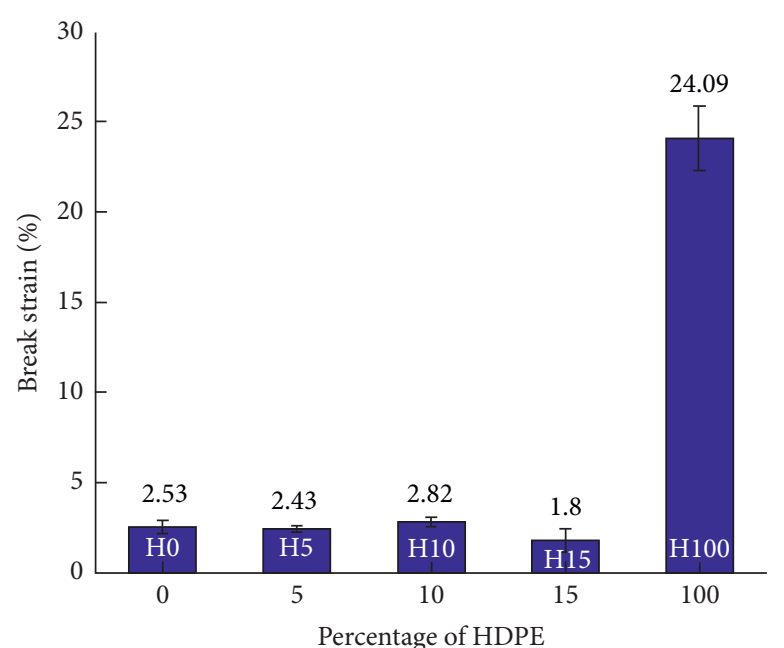

FIGURE 10: Effect of HDPE percentage on the break-strain value.

gradually increases and then gradually decreases as its ductility is much higher.

Figure 12 exhibits the effect of HDPE percentage on the flexural strength of the PBT/HDPE blends. The flexural strength of these samples is $87.79,70.47,55.3,58.98$, and $19.14 \mathrm{MPa}$ corresponding to $0 \%, 5 \%, 10 \%, 15 \%$, and $100 \%$ HDPE, respectively. Increasing the HDPE mostly results in decreasing the flexural strength.

The effect of HDPE percentage on the max-strain value is demonstrated in Figure 13. Overall, increasing the HDPE from 0 to $10 \%$ causes a minor change in max-strain values; the max-strain value slightly declines. This value obtains the lowest number of $4.50 \%$ at $15 \%$ HDPE, the same with $4.56 \%$ at $10 \%$ HDPE.

3.2. Izod Impact Test Results. PBT has good hydrocarbon barrier properties but has some obvious disadvantages such as low impact toughness [11]. Figure 14 indicates the effect of the HDPE content on the impact toughness of PBT/HDPE blends. The impact toughness of these samples is $2.70,2.52$, $2.65,2.59$, and $39.19 \mathrm{~kJ} / \mathrm{m}^{2}$ corresponding to $\mathrm{H} 0, \mathrm{H} 5, \mathrm{H} 10$, H15, and pure HDPE samples, respectively. Overall, adding 5-15\% HDPE does not cause much change in the impact toughness. Moreover, the impact toughness of the PBT/ HDPE mixtures is lower than that of pure HDPE. These results are consistent with the tensile strength results discussed in the previous section. These results also showed that an increase in the spherulite size of HDPE results in no improvement in the impact strength [13]. Wang et al. [5] studied HDPE and HDPE/PBT 80/20, and the results of impact strength showed that HDPE hardly improves the toughness of these blends and the impact value of the HDPE/ PBT mixture is $7.05 \mathrm{~kJ} / \mathrm{m}^{2}$, lower than pure HDPE. Besides, similar to the break-strain value as shown in Figure 10, Figure 14 presents a big variation due to the sudden change in the percentage of the PBT/HDPE mixture. The HDPE percentage has a rapid change from $15 \%$ to $100 \%$ of the mixture; therefore, the structure and the mechanical properties have a great change. 


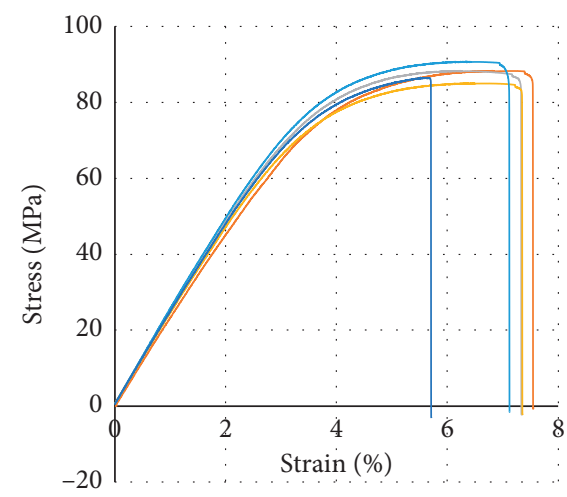

(a)

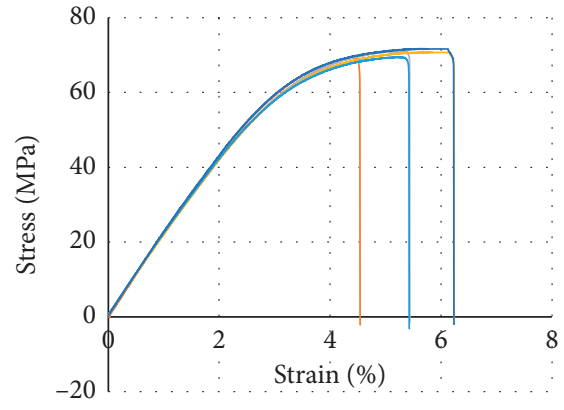

(b)

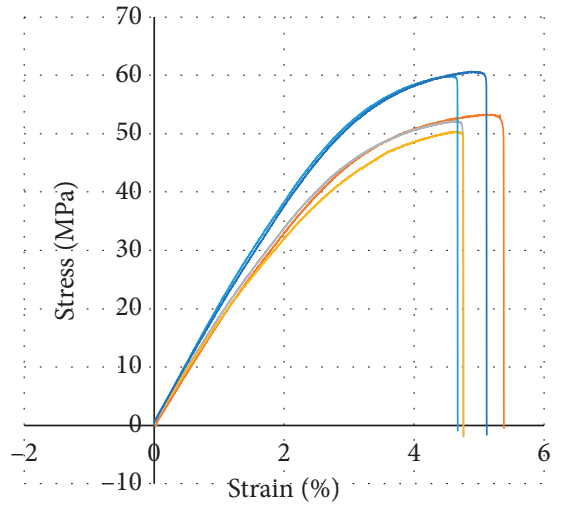

(c)

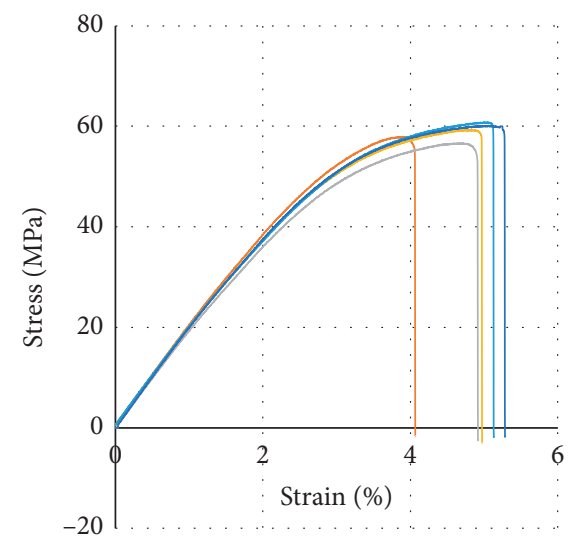

(d)

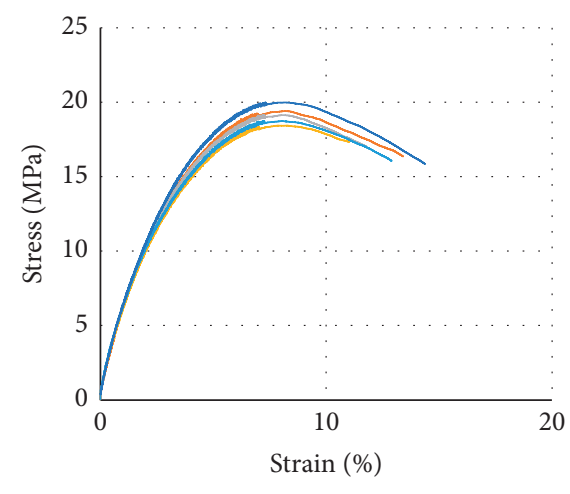

(e)

Figure 11: The stress-strain curves of the flexural test of the PBT/HDPE blends: (a) 0\% HDPE, (b) 5\% HDPE, (c) 10\% HDPE, (d) 15\% HDPE, and (e) 100\% HDPE.

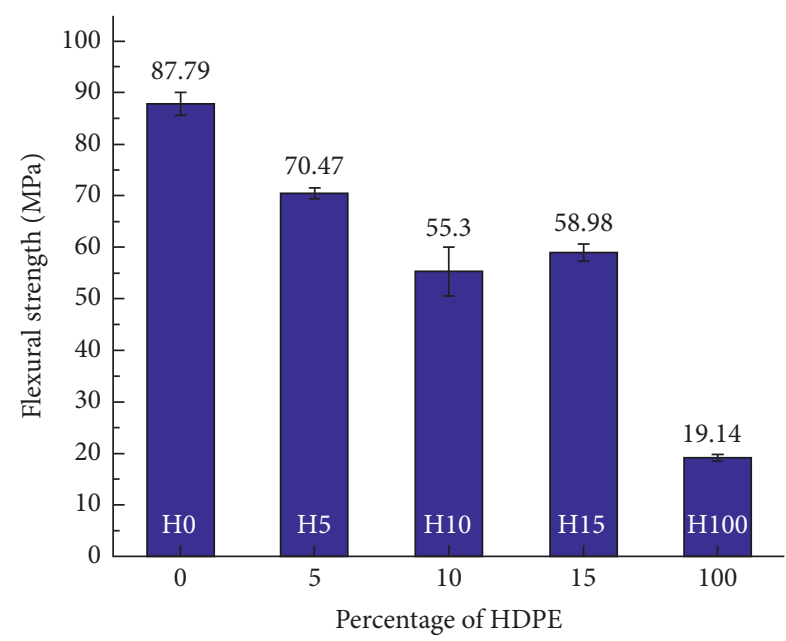

FIGURE 12: Effect of HDPE percentage on the flexural strength of the PBT/HDPE blends.

3.3. Microstructure Results. The properties of the polymer mixture are strongly influenced by its morphology. Figure 15(a)-15(c) show the SEM images of the PBT/HDPE mixture with different HDPE contents at a magnification of

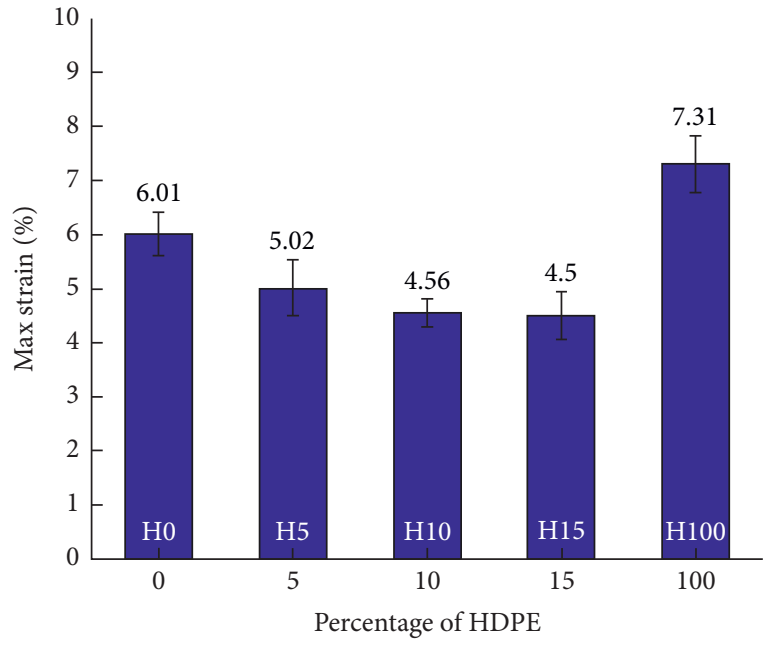

FIgURE 13: Effects on max-strain in different compositions of HDPE.

5000x. The pure PBT shows a relatively smooth surface, a characteristic of typical brittle fracture, as shown in Figure 15(a). By adding 5-15\% HDPE to the mixtures, the surfaces show more surface evidence of small fibers, as 


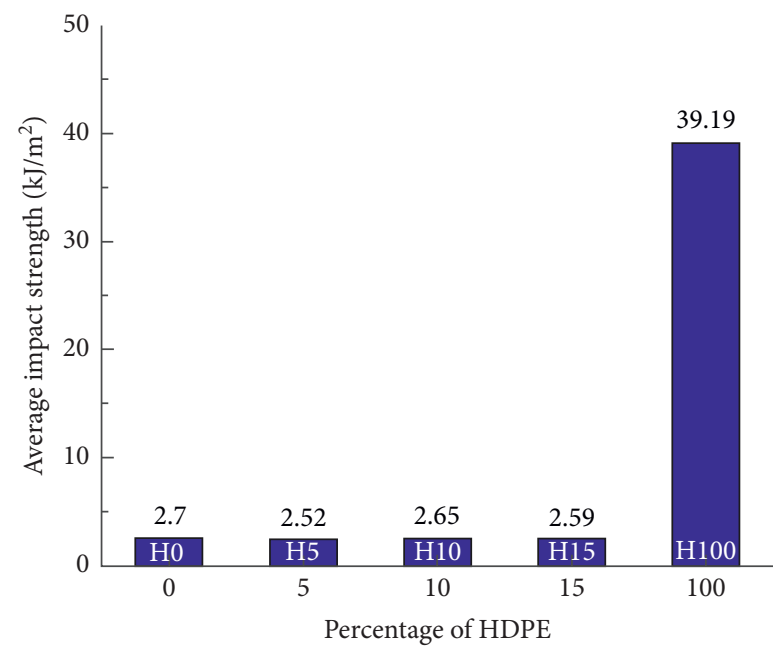

Figure 14: Effect of HDPE percentage on the impact toughness of the PBT/HDPE blends.

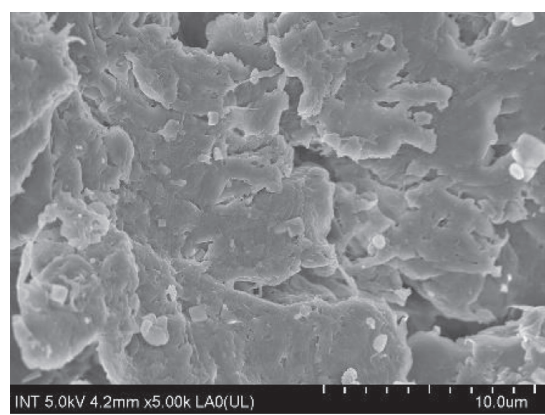

(a)

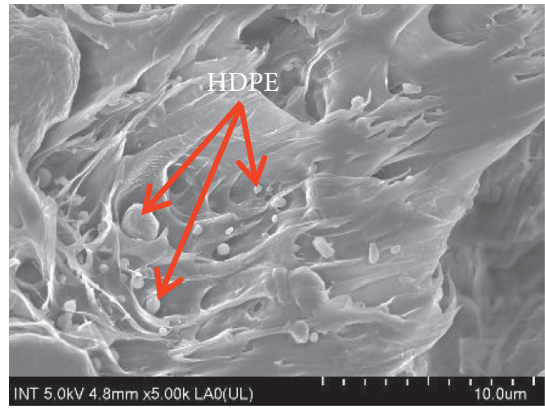

(d)

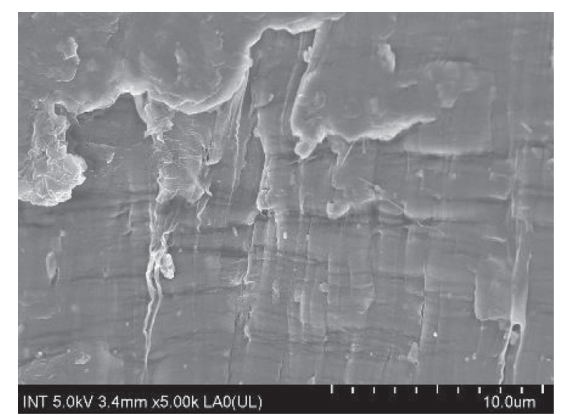

(b)

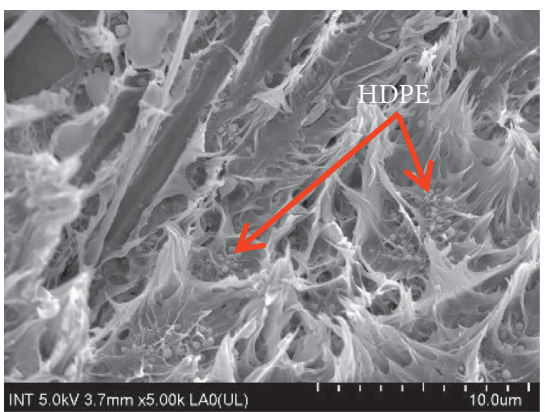

(c)

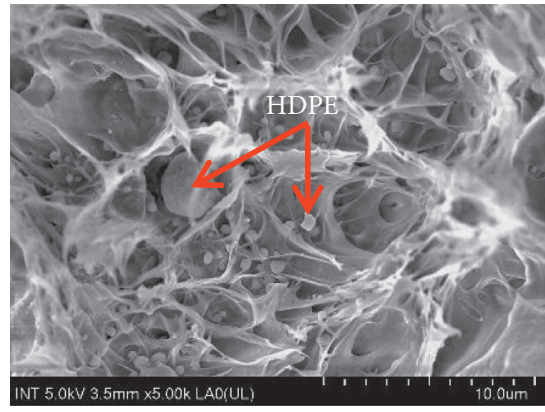

(e)

FIGURE 15: SEM micrographs of the PBT/HDPE blend with different weight percents of HDPE (5000× magnification): (a) 0\% HDPE (100\% PBT), (b) 100\% HDPE, (c) 5\% HDPE, (d) 10\% HDPE, and (e) 15\% HDPE.

shown in Figure 15(b)-15(d). Notably, Figure 15(b)-15(d) show that the spherical HDPE particles are scattered in the PBT matrix, representing a well-dispersed microstructure. Furthermore, there is no evidence of adhesion between the HDPE phase and substrate, as the surface of the HDPE particles is completely clear. This is the reason why the $5-15 \%$ HDPE samples do not present an improvement in the mechanical characteristics.

PBT and HDPE have a clear spherical crystal structure $[10,12]$. Figure 16(a)-16(c) show the dispersion of fine spherical particles of HDPE in a continuous matrix of PBT at a magnification of 30000x. Large cavities can be seen due to the removal of HDPE particles. Because the chemical structures of PE (nonpolar) and PBT (polar) are completely different, the two phases are seen directly on the microscope image. Incompatibility between PE and PBT can also be seen directly on the SEM image. Pesneau's study showed HDPE/PBT incompatibility because the surface of the PBT particles was completely clean, and the fault was always present at the interface [12]. Due to the lack of interphase adhesion, the mechanical properties of these mixtures are relatively poor. 


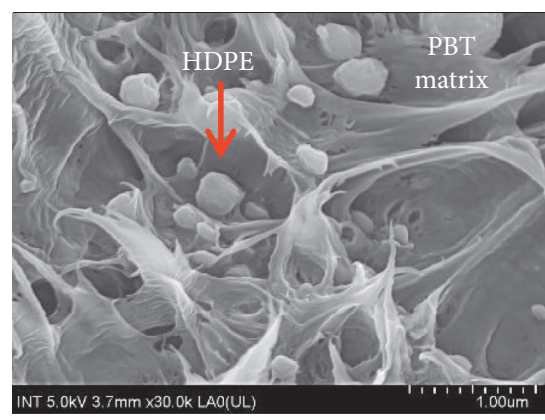

(a)

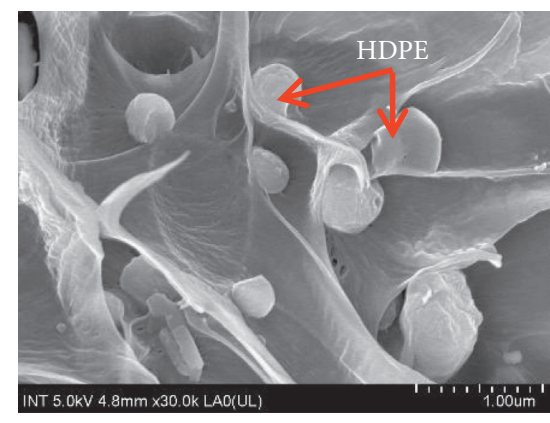

(b)

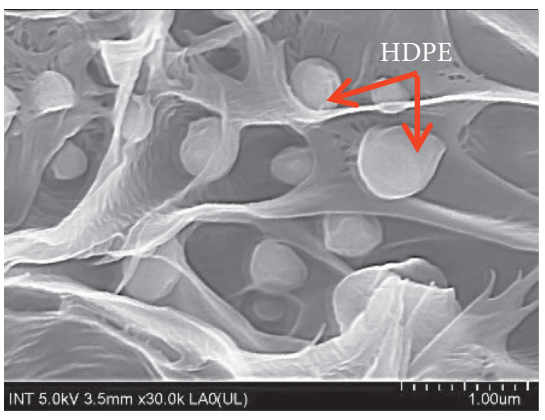

(c)

FIGURE 16: SEM micrographs of the PBT/HDPE blend with different weight percents of HDPE (30000× magnification): (a) 5\% HDPE, (b) 10\% HDPE, and (c) 15\% HDPE.

An increase in HDPE content leads to a further increase in the mean particle size. For a mixture containing $0 \%, 10 \%$, and 20\% HDPE, two distinct zones are clearly visible due to PBT and HDPE. Joung Sook Hong's study also demonstrated that the material with lower impact toughness forms a coating around the PBT core. This result also showed that a significant increase in the spherulite size of HDPE resulted in no improvement in the impact strength of HDPE/PBT [13].

\section{Conclusions}

(i) The tensile strength results show that the PBT/ HDPE mixture can present plastic damage. Adding HDPE will reduce tensile strength compared to pure PBT. 5\%, 10\%, and 15\% HDPE/PBT samples have the values $40.23,38.11$, and $27.77 \mathrm{MPa}$, respectively. These values are lower than that of pure PBT but still higher than that of HDPE.

(ii) Increasing the HDPE mostly results in a decrease in flexural strength. The flexural strength of these samples is $87.79,70.47,55.3,58.98$, and $19.14 \mathrm{MPa}$ corresponding to $0 \%, 5 \%, 10 \%, 15 \%$, and $100 \%$ HDPE, respectively.

(iii) Adding 5-15\% HDPE does not cause much change in the impact toughness. The impact toughness of the PBT/HDPE mixtures is lower than that of pure HDPE. The impact toughness of these samples is $2.70,2.52,2.65,2.59$, and $39.19 \mathrm{~kJ} / \mathrm{m}^{2}$ corresponding to $100 \% \mathrm{PBT}, 5 \%, 10 \%, 15 \% \mathrm{HDPE} / \mathrm{PBT}$, and pure HDPE samples, respectively.

(iv) The SEM microstructure of PBT and HDPE indicates a two-phase heterogeneous mixture with little or no adhesion between these phases.

(v) Further studies should be conducted to identify these morphological changes to the properties and also to correlate the morphology.

\section{Data Availability}

The data used to support the findings of this study are available from the corresponding author upon request.

\section{Conflicts of Interest}

The authors declare that there are no conflicts of interest regarding the publication of this paper.

\section{Acknowledgments}

The authors acknowledge the HCMC University of Technology and Education, Dong Nhan Phat Co., Ltd., and Material Testing Laboratory (HCMUTE). They gave the authors an opportunity to join their team and accessed the laboratory and research machines. Without their appreciated support, it would not be possible to conduct this research.

\section{References}

[1] B. S. Metwally, A. A. El-Sayed, E. K. Radwan, A. S. Hamouda, M. N. El-Sheikh, and M. Salama, "Fabrication, characterization, and dye adsorption capability of recycled modified polyamide nanofibers," Egyptian Journal of Chemistry, vol. 61, no. 5, pp. 867-882, 2018.

[2] F. M. Mwania, M. Maringa, and K. van der Walt, "A review of methods used to reduce the effects of high temperature associated with polyamide 12 and polypropylene laser sintering," Advances in Polymer Technology, vol. 2020, Article ID 9497158, 11 pages, 2020.

[3] D. Jubinville, B. P. Chang, J.-M. Pin, A. K. Mohanty, and M. Misra, "Synergistic thermo-oxidative maleation of PA11 as compatibilization strategy for PA6 and PBT blend," Polymer, vol. 179, p. 121594, 2019.

[4] S. Greiner, K. Wudy, L. Lanzl, and D. Drummer, "Selective laser sintering of polymer blends: bulk properties and process behavior," Polymer Testing, vol. 64, pp. 136-144, 2017.

[5] B. Wang, D. Wu, L. Zhu, Z. Jin, and K. Zhao, "High-density polyethylene-based ternary blends toughened by PA6/PBT core-shell particles," Polymer-Plastics Technology and Engineering, vol. 56, no. 17, p. 1908, 2017.

[6] A. Chalabi Tehran, K. Shelesh-Nezhad, K. Pouya Faraji et al., "A study of the effect of TPU and clay nanoparticles on the mechanical behavior of PBT-based nanocomposites," $\mathrm{Me}$ chanics of Advanced Composite Structures, vol. 4, pp. 179-186, 2017.

[7] G. V. Salmoria, V. R. Lauth, M. R. Cardenuto, and R. F. Magnago, "Characterization of PA12/PBT specimens 
prepared by selective laser sintering," Optics \& Laser Technology, vol. 98, no. 1, pp. 92-96, 2018.

[8] H. Yanping, H. Yang, H. Zhang, and Z. Mo, "Miscibility, crystallization behaviors and toughening mechanism of poly (butylene terephthalate)/thermoplastic polyurethane blends," Fibers and Polymers, vol. 19, no. 1, pp. 1-10, 2018.

[9] M. A. Mikitaev, G. V. Kozlov, A. K. Mikitaev, and G. E. Zaikov, "The Composite Models Application for Elastic Modulus of Poly(Ethylene Terephthalate)/Poly(Butylene Terephthalate) Blends Description," Bulletin of the Volgograd State University. Series 10. Innovative activity, vol. 18, no. 5, 2015.

[10] A. Hajibaba, M. Masoomi, and H. Nazockdast, "Compatibilization effectiveness of maleated polypropylene compared to organoclay in PBT/PP blends," Iranian Polymer Journal, vol. 25 , no. 2, pp. 157-167, 2016.

[11] W. Ignaczak, X. Sui, I. Kellersztein, H. D. Wagner, and M. El Fray, "The effect of fibre sizing and compatibilizer of polypropylene/poly (butylene terephthalate) blends on the mechanical and interphase properties of basalt fibre reinforced composites," Polymer International, vol. 67 , no. 4, pp. 414-421, 2018.

[12] I. Pesneau, P. Cassagnau, and A. Michel, "Morphology monitoring of PE/PBT blends by reactive processing," Journal of Applied Polymer Science, vol. 82, no. 14, pp. 3568-3577, 2001.

[13] J. Sook Hong, J. Lim Kim, K. Hyun Ahn, and S. Jong Lee, "Morphology development of PBT/PE blends during extrusion and its reflection on the rheological properties," Journal of Applied Polymer Science, vol. 97, pp. 1702-1709, 2005. 